\section{The challenge of adopting generic medicines in specialised clinical areas}

\author{
Peter Sharott
}

Throughout my 40-year career in hospital pharmacy, the substitution of originatorbranded (proprietary) medicines by their generic alternatives has been accepted as custom and practice. In the UK, hospital drug and therapeutic committees have adopted policies that enable pharmacists to automatically substitute generic alternatives without the need to consult prescribers, even when the original prescription has been written using the brand name. This practice has worked well for the vast majority of drugs, with relatively few exceptions or problems. As a general rule, because of potential differences in bioequivalence, UK hospital pharmacists do not substitute branded modified release formulations with alternative products in line with British National Formulary recommendations.

Throughout the UK, there are national hospital contracts for both oral and injectable generic medicines which enable the adoption of strictly applied quality standards for labelling, packaging and patient information leaflets. Regrettably, within contract terms and conditions, it is not possible to specify the size, shape and colour of solid doses to achieve a consistent approach across manufacturers. These contracts generally last for two years and have the benefit of facilitating market and supply chain management within the hospital service. On the other hand, the vast majority of repeat medicines are prescribed by family doctors and dispensed by community pharmacists. In primary care, the automatic substitution of generic alternatives for medicines prescribed by brand name is not permitted. However, the majority of prescriptions are written generically, and patients may experience frequent changes in the source of their medicines which may cause confusion and uncertainty and lead to reduced adherence to their treatment regimens.

This is not intended to imply that the use of generic medicines is unacceptable; rather that pharmacists in hospitals and the community, need to be vigilant in pro-

Correspondence to Peter Sharott, Pharmaceutical Adviser, NHS England (London Region), Mezzanine Floor, Southside, 105 Victoria Street, London SW1E 6QT, UK; peter.sharott@btinternet.com viding support to their patients so that clinical risks associated with nonadherence are minimised. On financial grounds, as health budgets are reduced in real terms and demand for healthcare increases, the rapid adoption of generic medicines has to remain a high priority. The very substantial savings that accrue from the use of generics support a growing 'save to invest' culture which releases funding for new, high-cost medicines and other healthcare priorities.

The two papers on the use of immunosuppressive drugs in organ transplantation, which appear in this edition of the journal, ${ }^{1} 2$ clearly demonstrate that the introduction of generic alternatives in highly specialised clinical situations is not always a straightforward matter, especially when the drugs have a narrow therapeutic index. Both papers recognise the cost benefits of adopting new generic immunosuppressants but, rightly, set this in the context of ensuring that there is no additional risk to patients caused by transplant rejection. The paper from Johnston ${ }^{1}$ highlights a particular concern about the lack of bioequivalence between different generic formulations. This issue needs to be addressed by the regulatory authorities, as clinicians must be confident that switching patients between products does not create unacceptable risks. The second paper by Devaney et $a l^{2}$ gives a practical example of how the issue of substitution of biosimilars might be tackled.

The example of immunosuppressive drugs raises a particular question about the different approaches that may be required when prescribing generic medicines in patients who are new to treatment, as opposed to those that are treatmentexperienced. In order to maximise savings opportunities it would be difficult to justify a non-switching policy for experienced patients, especially where long-term treatment is involved. The relatively low proportion of new patients would not be sufficient to realise substantial financial benefits. In London, the introduction of generic immunosuppressive drugs for experienced patients has been achieved through the provision of additional funding to support a short-term need for additional diagnostic tests and clinic visits to ensure that there is no increased risk to patients from transplant rejection following a switch to another product. Similar schemes have been introduced to manage the use of branded generic erythopoietins and granulocyte colony stimulating factors. The success of these initiatives has been due to close cooperation and collaboration between hospital consultants, clinical and specialist procurement pharmacists, and commissioners. Responsibility for prescribing and supply remains with the hospital and involves a high uptake of direct supply to patients' homes.

Within the HIV specialism, a different set of issues has emerged with the increasing availability of generic antiretroviral drugs. Theoretically, the direct substitution of a generic alternative to the originator-branded product should not cause exceptional difficulties. Indeed, clinical concerns about bioequivalence do not seem to be a major factor, but the worry is that patients, many of whom have been stabilised on antiretroviral therapy for a long time, have undetectable virus, and thus, do not represent a cross-infection risk, may become destabilised by changes in their dispensed medicines, leading to the risk of loss of viral control. In the UK, HIV patients generally receive their antiretroviral medication from a hospital pharmacy or, increasingly, from a homecare supplier. Family doctors and community pharmacists are not involved in the process making it much easier to ensure that there is a consistent supply of medication from the same manufacturer.

In London, about $80 \%$ of patients receiving antiretroviral drug therapy are prescribed a fixed dose combination (FDC) product containing two or three drugs. For the most part, this enables patients to take once daily dosage regimens, usually involving no more than two or three tablets. Clinicians strongly believe that once a day, as opposed to twice a day, regimens improve patient acceptability and, thus, adherence and that a singletablet regimen will have additional benefits, but the evidence for this is still inconclusive. This poses a particular challenge when considering the potential for switching patients from FDCs to regimens containing separate drugs in order to maximise the use of generics and the consequential financial benefits.

Clearly, there is an important role for hospital pharmacists in ensuring that patients receive appropriate support when they first receive a generic medicine. This could be achieved through either the provision of detailed written information or a face-to-face consultation at the next clinic visit, or a combination of both. In the UK, 
the HIV Pharmacy Association has recently been working on a set of standards for the switching of patients to generic antiretrovirals that are dispensed both by hospital pharmacies and homecare supply companies. This focused approach to the effective management of patients demonstrates the vital contribution that hospital pharmacists can make to balancing clinical and cost effectiveness with the needs and safety of individual patients.

Competing interests None.

Provenance and peer review Commissioned; internally peer reviewed.

To cite Sharott P. Eur J Hosp Pharm 2013:20:264-265.
Eur J Hosp Pharm 2013:20:264-265.

doi:10.1136/ejhpharm-2013-000383

\section{REFERENCES}

1 Johnston $A$. Equivalence and interchangeability of narrow therapeutic index drugs in organ transplantation. Eur J Hosp Pharm 2013;20:302-7.

2 Devaney A, Lee M. The use of generic immunosuppression for transplant patients: a UK perspective. Eur J Hosp Pharm 2013;20:272-4. 